\title{
The role of reconsolidation and the dynamic process of long-term memory formation and storage
}

\author{
Cristina M. Alberini ${ }^{1,2 *}$ \\ Department of Neuroscience, Mount Sinai School of Medicine, New York, NY, USA \\ 2 Department of Psychiatry, Mount Sinai School of Medicine, New York, NY, USA
}

Edited by:

Jacek Debiec, New York University, USA

\section{Reviewed by:}

Yadin Dudai, The Weizmann Institute of Science, Israel

Jacek Debiec, New York University, USA

\section{${ }^{*}$ Correspondence:}

Cristina M. Alberini, Department of Neuroscience, Mount Sinai School of Medicine, Box 1065, New York, NY 10029, USA.

e-mail: cristina.alberini@mssm.edu
It is becoming increasingly clear that the processes of memory formation and storage are exquisitely dynamic. Elucidating the nature and temporal evolution of the biological changes that accompany encoding, storage, and retrieval is key to understand memory formation. For explicit or medial temporal lobe-dependent memories that form after a discrete event and are stored for a long time, the physical changes underlying the encoding and processing of the information (memory trace or engram) remain in a fragile state for some time. However, over time, the new memory becomes increasingly resistant to disruption until it is consolidated. Retrieval or reactivation of an apparently consolidated memory can render the memory labile again, and reconsolidation is the process that occurs to mediate its restabilization. Reconsolidation also evolves with the age of the memory:Young memories are sensitive to post-reactivation disruption, but older memories are more resistant. Why does a memory become labile again if it is retrieved or reactivated? Here I suggest that the main function of reconsolidation is to contribute to the lingering consolidation process and mediate memory strengthening. I also discuss the literature and results regarding the influence of the passage of time on the reconsolidation of memory. These points have important implications for the use of reconsolidation in therapeutic settings.

Keywords: memory, consolidation, reconsolidation, molecular mechanisms

\section{THE DYNAMIC PROCESSES OF MEMORY CONSOLIDATION AND RECONSOLIDATION}

To become long-term memory, newly learned information undergoes progressive changes and a stabilization process known as memory consolidation (McGaugh, 2000; Dudai, 2004). This term was proposed more than 100 years ago by Muller and Pilzecker (1900) to indicate that, after learning, memory is initially in a labile state but, over time, becomes stable and resistant to disruption. Since then, many studies in different species and with numerous types of learning have shown that several kinds of interference, including blockade of new RNA and protein synthesis, disruption of the expression or function of specific proteins but also new learning, brain cooling, seizure, brain trauma, and brain regional lesions can disrupt the consolidation of the new memory.

Although all these treatments disrupt the formation of a new memory, the different types of interference revealed the existence of distinct temporal phases or processes that take place during memory consolidation. Molecular interference with protein and RNA synthesis inhibitors, blockers of receptors, kinases, neurotrophins, and transcription factors in species ranging from invertebrates to mammals showed that the biosynthetic-dependent phase of memory consolidation is relatively brief and is completed within the first day or two after training (Taubenfeld et al., 2001a,b; Bailey et al., 2004; Bekinschtein et al., 2007; Ou et al., 2010). Several reviews provide comprehensive summaries of what is known about the gradients of new protein and RNA synthesis requirements, as well as the nature of genes and proteins that are critically recruited during memory consolidation (Kandel, 2001; Davis and Laroche, 2006; Reissner et al., 2006; Wang et al., 2006; Bramham et al., 2010).
Proteins whose expression is induced by training and are required for long-term memory consolidation include several immediate early genes, such as the transcription factor CCAAT enhancer binding proteins (C/EBPs), c-Fos, and Zif268, as well as effector genes such as activity-regulated cytoskeletal protein (Arc) and tissueplasminogen activator (TPA), to mention just a few (Melchor and Strickland, 2005; Alberini, 2009).

On the other hand, animal model and clinical studies based on retrograde and anterograde amnesia caused by brain trauma, stroke, or brain region inactivation or ablation have shown that, in some types of memories such as the so-called explicit or declarative memories, consolidation lingers for a prolonged period. These memories engage the medial temporal lobe, and particularly the hippocampus and related structures, including the fornix and entorhinal cortex, for their consolidation. Hippocampal damage impairs human declarative memories that are several years old and animal contextual memories that are up to 30 days old; older memories remain unaffected (Smith and Squire, 2009). Thus it has been proposed that the hippocampus initially works with the neocortex to consolidate memory but, over time, gradually becomes less critical. In contrast, changes in the neocortex over time become more uniquely essential for storing the information by increasing connectivity among distributed cortical areas (Squire, 2009). Given the very different temporal scales of memory fragility in response to different types of interference, one mediated by gene expression during the first $1-2$ days after training and the other mediated by trace rearrangement among brain regions, it is unclear whether a relationship exists between the two temporal mechanisms of consolidation. It is, in fact, possible that the term consolidation is 
used in different settings and by different disciplines just to indicate memory maturation processes. Thus, it would be important to determine whether or not different types of interferences and amnesia gradients have mechanistic commonalities, and whether or not the terminology is the only common ground. As mentioned below, it is, however, intriguing to note that the reconsolidation sensitive period seems to overlap with the trace rearrangement consolidation temporal evolution.

Despite this question remains to be addressed, for several decades it was believed that memory consolidation is a unitary process: a newly formed memory requires gene expression for several hours, after which it becomes stable or consolidated (Davis and Squire, 1984). However, studies done in the last decade inspired by findings reported earlier, starting from the 1960s, demonstrated that the initial gene expression-dependent phase required for memory consolidation is not the only one. In fact, if memories that have become resistant to inhibitors of gene expression are retrieved, they again become labile for a limited time. During this time, the labile memory can be disrupted by similar gene expression blockers. The process, whereby a retrieved or reactivated labile memory re-stabilizes over time, is known as memory reconsolidation (Nader et al., 2000a; Sara, 2000a). A report by Nader et al. (2000b) awakened the scientific community to the retrieval-dependent fragility of memory. These authors reported that an established auditory fear conditioning memory, 24 h or 2 weeks after its encoding, when it generally is not sensitive to inhibition of new protein synthesis, again becomes transiently sensitive if it is retrieved. Since then, many studies in several species, and with respect to different types of learning, have reported similar conclusions proving the generalization of memory reconsolidation (Dudai and Eisenberg, 2004; Alberini, 2005, Tronson and Taylor, 2007; Nader and Einarsson, 2010).

These results also led to many questions: Is reconsolidation a recapitulation of the initial consolidation process? Do memories undergo reconsolidation every time they are retrieved? Do all types of memories undergo reconsolidation? What is the relationship between reconsolidation and extinction? What mechanisms underlie reconsolidation? What is the function of reconsolidation? Is the age of a memory a critical variable for reconsolidation? Can we target the reconsolidation process to weaken memories that contribute to diseases such as post-traumatic stress disorder (PTSD) and addiction?

In this article, I will focus on the last three questions, then propose a model of memory reconsolidation.

\section{THE FUNCTIONS OF MEMORY RECONSOLIDATION}

Why does memory become labile after retrieval? It is important to answer this question, not only because it will allow a better understanding of how the memory process works, but also because this knowledge is essential to developing accurate clinical approaches that will use post-retrieval strategies to disrupt or weaken pathogenic memories. At least two, non-mutually exclusive hypotheses have been proposed to explain the function of reconsolidation. According to one, memory becomes labile because through reconsolidation new information is integrated into the background of the past, thus allowing the memory to be updated (Lewis, 1979; Sara, 2000a; Dudai, 2004). The other proposes that memory reconsolidates in order to become stronger and longer lasting (Sara, 2000b).
The first hypothesis has been tested by several studies in various species. The conclusions reached are contradictory. Some studies state that reconsolidation does not mediate memory updating, others that it does. This controversy seems to be due in part to the fact that the same term was used to indicate different types of behavioral updating, and in part to conflicting interpretations of the results. In general terms, we can define memory updating as all changes that are incorporated into a reactivated memory due to current perceptual input. Thus, every time a memory is retrieved it undergoes changes because, in fact, it is perceived in a different moment and with different current inputs. This implies that both a distinct experience that becomes linked to a first, reactivated memory, as well as a second identical learning experience, can be defined as memory updating. However, it is obvious that these two conditions represent very different updating processes. One has to do with how distinct experiences, that is, two distinct traces, become linked or associated; the other has to do with how the same learning experience adds to a first one by repetition.

The studies that tested whether reconsolidation mediates updating after the reactivation of the same experience (e.g., multiple training trials) need to carefully define the use of the terms consolidation and reconsolidation in those behavioral paradigms. Moreover, an important caveat should be kept in mind when testing the differential role of reactivated or new traces: that both consolidation and reconsolidation are sensitive to many of the same interfering agents, including protein synthesis inhibitors, pharmacological and molecular interferences, and new learning. Thus, a result showing that both the old and updated traces are disrupted by one treatment does not prove that reconsolidation mediates updating. In order to dissect whether reconsolidation differentially contributes to the updating of memory, it is necessary to use interfering approaches that selectively disrupt either reconsolidation of a reactivated memory or the consolidation of new traces.

A factor that has been considered critical to the definition of memory reconsolidation is whether amnesic agents applied after memory reactivation disrupt the original memory trace or whether reactivation, by definition, produces a new memory that undergoes consolidation. If reactivation produces a new trace, then disrupting this trace should not affect the old memory; there should be no decrease in memory retention below the levels reached by the old memory (Debiec et al., 2002). Reconsolidation experiments in many different tasks and species have shown that this is not the case. To the contrary, it has been established that, after retrieval or reactivation, the old trace is indeed, in significant part, sensitive to disruption. However, although this argument is clear with reactivations evoked by an experience that is different from the original training, for example a non-reinforced reminder after a reinforced conditioning, it is confusing in the case of the contribution of repeated training trials. Should it be called consolidation or reconsolidation the process induced by a second learning trial that, if challenged with an amnesic agent, results in memory disruption that goes below the retention level produced by the first trial?

I will review some of the results and then return to these general issues about defining consolidation and reconsolidation.

In my laboratory, we focused on testing an updating produced by linking two different experiences (Tronel et al., 2005). This study asked whether linking new information to a previously 
established memory via its reactivation critically depends on the reconsolidation of this memory. In other words, does reconsolidation mediate the incorporation of new information? To address this question, we used an inhibitory avoidance task, modified to a second-order conditioning paradigm and the anatomically distinct requirement of the transcription factor $\mathrm{C} / \mathrm{EBP} \beta$, specifically in hippocampus but not amygdala during consolidation and in amygdala but not hippocampus during reconsolidation. Secondorder conditioning promotes the formation of associations between a new conditioned stimulus (CS2) and a conditioned response elicited by another CS (CS1) that was previously associated with an unconditioned stimulus (US; Rescorla, 1980; Gewirtz and Davis, 2000). Thus, the stimulus-response learning that occurs during second-order conditioning represents the formation of an association between new (CS2) and reactivated information (memory of CS1-US), which makes this paradigm proper for investigating the mechanisms involved in linking new to reactivated information. We found that linking new information to a reactivated memory is not mediated by the reconsolidation of the reactivated memory. In fact, when we selectively disrupted the reconsolidation of the CS1-US, the second-order CS2-(CS1-US) remained intact. In contrast, when we blocked consolidation selectively, only the new linking (the update) was disrupted, whereas the original memory remained intact. We concluded that when two distinct experiences, one reactivated and one new, become linked, the reconsolidation of the reactivated trace does not mediate this updating (Tronel et al., 2005). In agreement with this result, Debiec et al. (2006), using rat auditory fear conditioning have found that disrupting the reconsolidation of CS1-US by inhibiting amygdala protein synthesis after reactivation does not affect the association CS2-CS1-US. Results similar to ours were found with associative memories in the Crab Chasmagnatus (Suárez et al., 2010), as well as in humans (Forcato et al., 2010). Hence, the function of reconsolidation must be different from that of linking a reactivated memory to a novel, distinct experience. In general terms, we can infer that memory updating via formation of complex networks requires memory retrieval, but not reconsolidation and that the reconsolidation of a reactivated memory does not alter the entire network of updated associative memories.

Other studies tested whether reconsolidation mediates memory updating by examining the contribution of the repetition of similar training trials or retrievals as compared to that of new encodings. These studies concluded that with multiple learning trials or reactivations, memory does indeed become labile. However, the fragility is seen only when learning is in a non-asymptotic mode. When memory has reached an asymptotic level, it is resistant to disruption by post-reactivation amnesic treatments, but then becomes labile again if it is in a new encoding mode (Rodriguez-Ortiz et al., 2005; Morris et al., 2006). For example, Morris et al. (2006), using rat learning to locate a platform in a water maze concluded that: "the engagement of a memory-encoding mode during the act of spatial memory retrieval may be one requirement for reconsolidation to be observed. This occurs in spatial tasks when animals are confronted by spatial novelty or 'mismatch' that triggers exploration and the updating of their cognitive representation of space" and proposed that a dual encoding and retrieval state is required for the reconsolidation of spatial memory. Similar conclusions were offered by
Rodriguez-Ortiz et al. (2005). However, an alternative interpretation of the data, is that when new encoding is presented after the reactivation of a memory, two traces exist and are both in a labile state: one that mediates the reconsolidation of the reactivated trace if in a non-asymptotic phase and another that represents the newly encoded trace which may undergo a new consolidation process. Since the results of these studies do not clearly dissect the contribution of reconsolidation of an established memory versus consolidation of a new trace, they do not conclusively demonstrate a role for reconsolidation in the new encoding. Nevertheless, it is interesting to note that inhibition of protein synthesis after repeated trials during a non-asymptotic phase returns the memory performance to the pretraining chance level, suggesting that the original trace had remained labile. Similar results have been reported in other studies that used multiple trial learning tasks to investigate the effects of amnesic treatments on memory consolidation (e.g., Meiri and Rosenblum, 1998; Luft et al., 2004; Touzani et al., 2007).

Conclusions similar to those of Morris et al. (2006) were offered by Winters et al. (2009), who used object recognition memory. As in the previous studies, it is unclear whether the disruption of the memory (object recognition, in this case) occurs because, in the so-called "reactivation" trial, the rat associates the old information (the objects) with a novel environment and the MK-801 disrupts consolidation of this new memory trace, which, in fact, includes the old objects.

Thus, my interpretation of these and other studies based on multiple learning trials, including attenuation of neophobia, incentive instrumental learning, and the Morris water maze (Hernandez et al., 2002; Rodriguez-Ortiz et al., 2005, 2008; Morris et al., 2006; Rossato et al., 2006; García-DeLaTorre et al., 2009), is twofold. First, in agreement with the authors' conclusions, during a learning curve, post-trial applications of amnesic treatments disrupt memory retention only when the memory is not in an asymptotic level. However, when retention has reached an asymptotic level and no further learning or increased retention is evident, a previously consolidated memory remains stable and resistant to disruption. Second, if then new events are presented and associated with this memory, a new trace is formed which is labile because it undergoes consolidation. If part of the old information is incorporated in a new trace, its retention might be disrupted if the new trace is challenged by amnestic treatments, following the rules of the predominant active trace (Eisenberg et al., 2003).

Perhaps there is the need to revisit or clarify some terminology. Should the process of fragility and restabilization resulting from an identical learning trial that is in a non-asymptotic phase of learning be called consolidation or reconsolidation? Is the distinction between consolidation and reconsolidation related to the level of memory disruption (whether is goes below the incremental level)?

If one defines reconsolidation as the process that occurs after each sequential trial during the non-asymptotic phase of a learning curve, then the classic definition of the consolidation process of a multiple trials task coincides with what here would be called a process of consolidation-reconsolidation. Interestingly, this stabilization, which would occur through rounds of reconsolidation and result in a memory resilient to disruption, is highly reminiscent of that found after a salient single-trial learning, which over time develops a graded increased resistance to reconsolidation 
disruption (see below). This view of reconsolidation as an integral part of the consolidation process was proposed a few years ago by both Dudai and Eisenberg (2004) and myself (Alberini, 2005). In other words, it seems that reconsolidation, as part of a lingering consolidation process, strengthens memory retention by following the rules of trace dominance (Eisenberg et al., 2003). Notably, what the classical consolidation hypothesis did not account for, nor explored, are the mechanistic contributions of each reactivation of the trace that can be elicited by each learning trial, or the fact that non-reinforced reminders can produce a similar result.

Supporting the conclusion that reconsolidation increases memory strength, Lee (2008) reported that a second learning trial strengthens a contextual fear memory. He then showed that when hippocampal injection of BDNF antisense oligodeoxynucleotide (ODN), which had been found to disrupt the initial consolidation but not reconsolidation of this memory (Lee et al., 2004), was given before the second trial, it did not change memory retention. However, injection of zif268 antisense, which had been shown to disrupt reconsolidation but not consolidation of contextual fear conditioning, when given before the second training trial, completely disrupted memory retention. Lee therefore concluded that reconsolidation mediates memory strengthening. His results are in agreement with several lines of evidence that memory reconsolidation, like strengthening (Lee, 2008), uses mechanisms that differ from those used during the initial wave of consolidation (Taubenfeld et al., 2001a; Lee et al., 2004; Tronel et al., 2005; von Hertzen and Giese, 2005). Recent results from our laboratory, obtained by using inhibitory avoidance in rats and non-reinforced reminders, lead to similar conclusions; we found that retrievals of a young memory, which are accompanied by its reconsolidation, result in memory strengthening and contribute to its overall consolidation and concluded that a function of reconsolidation is to mediate memory strengthening and prevent forgetting (Inda et al., 2011).

In agreement with this conclusion, earlier evidence indicated that, following reactivation, memory can indeed be strengthened. For example, memory reactivation through the re-experience of a single contingent-reinforcing stimulus given in the same context as previous intermediate-term memory training boosts a residual memory trace into long-term memory (Parvez et al., 2006). Electrical stimulation of the mesencephalic reticular formation (MRF) improves the retention of memories, including fear conditioning and linear maze, when the stimulus is applied after reactivation (DeVietti et al., 1973; Sara, 2000a). During the reconsolidation phase, memory retention can be increased by pharmacological modulation (Frenkel et al., 2005; Tronson et al., 2006), suggesting that after reactivation memory can be strengthened. Thus, memory enhancement, like memory disruption, can be achieved by targeting a post-retrieval or post-reactivation memory process. This opportunity to enhance memory strength following retrieval, like that of disrupting it, has important clinical implications.

In summary, based on the current knowledge, I suggest that the functional role of reconsolidation, whether induced by a second training trial or a non-reinforced reminder, is to mediate memory strengthening and prevent forgetting. In contrast, memory updating with new learning and encoding results in a new memory trace that is accompanied by a new consolidation process. This new and the old memory traces coexist. It seems likely that traces that are retrieved or reactivated will reflect which information will undergo reconsolidation and strengthening. Thus, the strengthening evoked by a second training trial will be qualitatively and, perhaps, quantitatively different from that evoked by a non-reinforced reminder. Understanding the functions and boundaries of the reconsolidation process is critical for designing treatments that aim at memory disruption or enhancement.

\section{MEMORY RECONSOLIDATION AND THE PASSAGE OF TIME}

A key question is: what happens to an encoded and consolidated memory over time? Time is an extremely important parameter in memory formation, allowing the selection of what will be stored as long-term memory and dictating what and for how long information will be stored. Memory consolidation evolves over time and memories change with time.

Clinical studies of retrograde amnesia inspired the concept of memory consolidation (Squire and Alvarez, 1995; McGaugh, 2000), according to which medial temporal lobe structures are necessary for the formation and maintenance of a memory for a limited time. During this time, there seems to be a reorganization of the memory trace. After this reorganization is completed, the medial temporal lobe structures are no longer essential for maintaining the information. Indeed, clinical and experimental evidence indicate that damage to the medial temporal lobe does not interfere with the storage of remote memories, but does impair recent memories in a temporally graded fashion. As mentioned earlier, the temporal window of this circuitry rearrangement seems to be approximately a few weeks in animal models and a few years in humans (Squire, 2009). In agreement with this temporal evolution of memory consolidation, the expression of activity-related genes such as c-Fos, as well as the uptake of 2-deoxyglucose induced by recall of contextual fear conditioning in rats decrease in the hippocampus and increase in cortical areas as the memory ages over several weeks (Frankland and Bontempi, 2005). Neuroimaging studies in humans have shown a similar anatomical distribution of activity when subjects recall memories ranging from 1 to 30 years old. Other support to this temporal frame comes from recordings from cell populations in the medial prefrontal cortex (mPFC) in rats during and after eyeblink conditioning. Moreover, firing-rate activity selective for memory associations starts to develop during the late stage of acquisition and continues for up to 6 weeks independently of whether or not the animals are continuously trained. This slow time course of firing-rate changes agrees with a previously observed time window in which the MPFC becomes important for retrieval. Finally, in line with the key principles of the consolidation theory, associative learning seems to initiate a temporally graded process that leads to long-lasting changes in cortical areas (e.g., the $\mathrm{mPFC}$ ), even without continued training (Takehara-Nishiuchi and McNaughton, 2008). Thus, there is little doubt that memory consolidation and storage evolve with time. It is possible, as indicated by animal studies, that the rearrangement of the memory representation from hippocampal to cortical areas, occurs together with a qualitative transformation; hence, initial memory, which would be highly detailed and context-specific may become one that is more general (Moscovitch et al., 2005; Winocur et al., 2007, 2010). 
How do these temporal changes affect memory reconsolidation? In other words, is the post-retrieval lability of memory a function of time? Understanding the temporal boundaries of reconsolidation is critical for the development of efficacious clinical approaches that target the reconsolidation process.

The initial hypothesis of memory reconsolidation proposed that, when a memory is active (following training or reactivation), it is in a labile state, but that when it is inactive, it is stable (Nader et al., 2000a). This implies that every time a memory is reactivated (active) it becomes labile. Many studies have tested the role of time in memory reconsolidation. The results have shown that, in most cases, in a variety of species, including rat, mouse, chick, and Medaka fish, the passage of time critically influences the stability of a memory, so that the older a memory becomes, the less susceptible it is to disruption following its reactivation. These studies report increasing resilience to disruption following memory reactivation over a time window of several weeks. For example, in rat inhibitory avoidance, 2- and 7-day-old memories are disrupted by protein synthesis inhibitors administered after retrieval, while 14- and 28-day-old memories are resistant to the same treatment (Milekic and Alberini, 2002). Similarly, in Medaka fish, the administration of a $\mathrm{Na}^{+}$channel blocker at the time of memory retrieval disrupts a 4-day-old memory and has an intermediate effect on a 9-day-old memory, but exerts no effect on a 15-day-old memory (Eisenberg and Dudai, 2004). Studies of other species and different types of learning, including both aversive and appetitive, have confirmed the existence of a temporal gradient of progressive resilience to memory disruption after reactivation (Litvin and Anokhin, 2000; Suzuki et al., 2004; Boccia et al., 2006; Frankland et al., 2006; Robinson and Franklin, 2010). Additional findings have shown that, in addition to the age of a memory, its strength and the reactivation intensity interact to influence the degree of vulnerability of a reactivated memory (Suzuki et al., 2004, Diergaarde et al., 2006; Bustos et al., 2009). For example, Suzuki et al. (2004) showed that whereas protein synthesis inhibitors can disrupt a reactivated 24 -h- to 3 -week-old contextual fear memory in mice, an 8-week-old memory remains unaffected by a similar reactivation. However, if the reactivation session is prolonged, even the older memory can be disrupted. Finally, weak ( 1 shock) versus strong (3 shocks) training protocols correlate, respectively, with more or less susceptibility to disruption after reactivation. It is important to keep in mind that memory changes over time and that long-lasting reactivation preferentially evokes extinction (a decrease in the conditioned response resulting from CS presentations) over reconsolidation (Eisenberg et al., 2003, Bustos et al., 2009). Thus, for stronger memories, which are insensitive to reconsolidation disruption but become sensitive when a long reactivation is applied, it is important to exclude the possibility that memory disruption is the result of a facilitated extinction.

The fact that, as noted earlier, memory changes over time is also supported by molecular experimental evidence, including reconsolidation studies. Reconsolidation has distinct requirements than the initial consolidation (Alberini et al., 2006). Reconsolidation of an associative memory in Lymnaea, which is evident at both 6 or $24 \mathrm{~h}$ after training by using protein synthesis inhibitors, shows different molecular requirements: only reconsolidation at $6 \mathrm{~h}$ after training, but not at $24 \mathrm{~h}$, requires protein kinase A activity. Hence, even seemingly consolidated memories undergo further selective molecular maturation processes (Kemenes et al., 2006).

In summary, over time, memory undergoes many molecular, anatomical, and qualitative changes and these include its increased resilience to reconsolidation disruption. Some authors disagree with this conclusion. They view reconsolidation as a general process that occurs following memory retrieval without temporal constraints, and explain temporal boundaries as experimental limitations (Nader and Einarsson, 2010). Nader et al. (2000b) and Debiec et al. (2002) showed that 2-day- and 2-week-old cuedfear conditioning memories, as well as a 45-day-old contextual fear conditioning memories in rats, are disrupted, respectively, by post-retrieval bilateral injection of anisomycin into the amygdala or hippocampus. In disagreement with these contextual fear conditioning results, other groups have reported that, with time, contextual fear memories in mice become less sensitive to post-retrieval interference (Suzuki et al., 2004; Frankland et al., 2006). Other memories have been reported to have a long-lasting temporal window of post-reactivation fragility. For example, a 21-day-old appetitive instrumental response (sucrose self-administration) is disrupted by systemic injection of the $\beta$-adrenergic antagonist propranolol, while a 27-day-old cocaine-induced conditioned place preference is disrupted by post-reactivation amygdala injections of zif268 antisense (Diergaarde et al., 2006; Lee et al., 2006). In contrast, studies on morphine-induced conditioned place preference show that increased resistance to post-reactivation treatment with propranolol or the GABA (A) agonist midazolam develops with stronger training (Robinson and Franklin, 2010).

These opposing results can be explained by the findings discussed earlier, which have shown that the nature of the learning task and therefore its underlying circuitry as well as the intensity of training and reactivation are all contributing to produce gradients of post-retrieval protein synthesis requirement that have different temporal evolutions. It is consistent with a lingering consolidation and the effects of retrograde amnesia on medial temporal lobedependent memories that the stabilization process may take a long time. Hypotheses that could be explored are whether the increased resilience to reconsolidation disruption is related to its increased cortical representation or increased distribution over brain areas (Nadel and Moscovitch, 1997), or the implicit or explicit nature of the memory and its storage distribution.

It is important that these issues be addressed because it will provide useful information for designing clinical trails.

Importantly, the existence of a gradient does not exclude the possibility that reactivation of fully consolidated memories or a memory in an asymptotic phase is accompanied by a phase of de novo protein synthesis. However, new molecular changes induced by the reactivation of a fully consolidated memory may occur without manifesting fragility of the consolidated information.

\section{POTENTIAL CLINICAL APPLICATIONS}

The findings on memory reconsolidation have triggered great interest in the medical community, particularly among mental health professionals who treat disorders that are based on pathogenic memories. Two pathologies that could benefit from targeting 
memory reconsolidation as a potential therapeutic approach are PTSD and drug addiction, both of which are characterized by the presence of strong, repeatedly recalled memories.

Post-traumatic stress disorder can develop after exposure to an emotionally or physically traumatic event. One hallmark of the disorder is that the subject keeps reliving the trauma through repeated, intrusive memories of the initiating experience. PTSD patients have difficulty sleeping and feel detached or estranged. These symptoms can be so severe and persistent that they significantly impair patients' ability to function. Although no animal model is yet available to reproduce PTSD fully, various behavioral paradigms have been used to represent some component of the disorder, using a rationale description of how PTSD develops. According to this description, the traumatic event (US) triggers a strong hormonal stress response, which mediates the formation of a robust and enduring memory of the trauma. Subsequent recall of the event in response to cues and reminders (CS) releases more stress hormones (conditioned response) and even further consolidates the memory, leading to PTSD symptoms such as flashbacks, nightmares, and anxiety (Pitman and Delahanty, 2005). The persistence of PTSD can be explained in terms of trauma-induced strengthening of the memory trace. For example, it is hypothesized that noradrenergic hyperactivity and stress hormones facilitate the encoding and consolidation of the memory (Pitman, 1989; O'Donnell et al., 2004; Yehuda, 2006). Thus, animal models that evoke strong fear conditioning memories and high-anxiety behavior have been used to study questions that are clinically relevant to PTSD.

In principle, the pharmacological and behavioral interferences thus far found to be effective in disrupting fear memory reconsolidation or enhancing extinction (not discussed in this paper) could potentially be useful for identifying new treatments that can be tested in clinical trials. Some of the compounds found to affect reconsolidation, including antagonists of $\beta$-adrenergic or glucocorticoid receptors (GRs), are already used in clinical pharmacology for treating other diseases. Hence, they are the most readily available potential therapies for targeting reconsolidation in PTSD and addiction.

Antagonists of the $\beta$-adrenergic receptor, such as propranolol, have already been explored at preclinical and clinical levels. Propranolol, which is most commonly used to treat hypertension, has been administered in concert with the retrieval of a fearful or traumatic event in both animals and humans. Przybyslawski et al. (1999) provided one of the first reports on the effect of propranolol on memory reconsolidation. Using rat inhibitory avoidance, these authors found that systemic administration of propranolol after the reactivation of an inhibitory avoidance memory disrupted the memory on subsequent tests. However, we recently reached the opposite conclusion. Because the shock used by Przybyslawski et al. (1999) was very weak (0.2 mA), we set out to determine whether the reconsolidation of a memory induced by a greater shock, which would more closely approximate a traumatic event, was sensitive to propranolol treatment. Hence, we tested the effect of the same propranolol treatment in rats given either before or after the retrieval of an inhibitory avoidance memory evoked by a 0.6 - or $0.9-\mathrm{mA}$ foot shock. We found that although these memories could be disrupted by several other treatments, like anisomycin or the GRs antagonist RU38486, propranolol had no effect (Muravieva and Alberini,
2010). These divergent results could be related to several differences in the protocols used. However, to provide solid preclinical information, it is important that the potency and generalization of the propranolol treatment on fear memories be established. Using another paradigm known as auditory fear conditioning, Debiec and LeDoux (2004) have found that propranolol injected either systemically or into the lateral nucleus of the amygdala after reactivation of a 1-day- or 2-month-old memory weakened the fear response when tested $48 \mathrm{~h}$ later. In agreement, in recent studies based on contextual-auditory Pavlovian fear conditioning, we found that systemic propranolol injection following a retrieval elicited by cue exposure interferes with the reconsolidation of both cued and contextual fear conditioning. On the other hand, propranolol administered after contextual reactivation only affects contextual fear conditioning and has no effect on the auditory fear. Thus, it seems that the efficacy of systemically administered propranol in disrupting the reconsolidation of fear memories might be limited (Muravieva and Alberini, 2010).

Other studies reported divergent results on the effect of propranolol on memory reconsolidation: Whereas the reconsolidation of eyeblink conditioning potentiation and conditioning to natural or drug-associated reward is disrupted by propranolol (Diergaarde et al., 2006; Milton et al., 2008; Kindt et al., 2009), the reconsolidation of appetitive Pavlovian memories in rats (Lee and Everitt, 2008) and neutral and emotional verbal memories in humans (Tollenaar et al., 2009) are not. Interestingly, declarative measures for the acquired contingency between the CS and US (Kindt et al., 2009) are insensitive to propranolol treatment, but the fear response is sensitive. Hence, as Kindt et al. (2009) have suggested, propranolol may target the fear response, but not the cognitive or explicit components of that response, and further studies should be able to address this question.

The glucocorticoid pathway is another pharmacologically targeted pathway for the potential treatment of PTSD. The endogenous stress hormone corticosterone bidirectionally modulates memory retention (McGaugh and Roozendaal, 2002; Roozendaal, 2002). Low doses increase memory retention, while high doses disrupt it. Recent studies have shown that glucocorticoids administered after the reactivation of a contextual fear memory have an amnesic effect on the original memory. These studies also have provided evidence that a possible mechanism for this effect is enhancement of extinction of the expression of the original memory (Cai et al., 2006). My laboratory investigated the effect of blocking the GRs on inhibitory avoidance memory reconsolidation in rats. We found that post-retrieval, intra-amygdala blockade of GRs by the antagonist RU38486 significantly disrupts inhibitory avoidance in rats (Tronel and Alberini, 2007). Further investigations have shown that also systemic administration of RU38486, either before or after retrieval, consistently weakens inhibitory avoidance retention in a dose-dependent manner. The efficacy of treatment appears to be a function of the intensity of the initial trauma; however, highly traumatic memories are disrupted by changing the time and number of interventions. Furthermore, we found that one or two treatments are sufficient to maximally disrupt the memory and that the treatment selectively targets the reactivated memory without interfering with the retention of another unreactivated memory (Taubenfeld et al., 2009). 
In light of these results, the glucocorticoid pathway appears to be a promising site of pharmacologic intervention for trauma-related pathologies, including PTSD.

A novel and very interesting alternative to pharmacological disruption, which uses a behavioral design, has recently been provided by animal and human studies based on sequential retrieval (reconsolidation) and extinction. Extinction training after fear conditioning retrieval leads to permanent memory impairment if it is presented within a post-retrieval reconsolidation temporal window (Monfils et al., 2009; Schiller et al., 2010). Further studies should be able to elucidate the underlying mechanisms of this interesting approach.

Another pathology that can take advantage of reconsolidation studies is addiction. Substance abuse generally leads to a chronic condition believed to result from an addict's inability to permanently abstain from drug use. Drug addicts repeatedly relapse to drug seeking even after years of abstinence. This pathologic behavior is frequently induced by the recall of memories and environmental stimuli that are intimately connected to the rewarding effects of the drug (O'Brien et al., 1992). Therefore, disruption of memory reconsolidation provides an unprecedented potential strategy to disrupt memories that facilitate drug addiction. Promising results have recently been achieved in animals dependent on morphine or cocaine by injecting, after memory reactivation, inhibitors of protein synthesis, extracellular signal-regulated kinase (ERK), or $\beta$-adrenergic receptors, or by disrupting the expression of the immediate early gene zif268 either peripherally and within specific brain regions, such as the amygdala, hippocampus, or nucleus accumbens (Lee et al., 2005; Miller and Marshall, 2005; Milekic et al., 2006; Valjent et al., 2006; Fricks-Gleason and Marshall, 2008; Robinson and Franklin, 2010). In some of these studies, inhibitors were injected in animals that had acquired a place preference in response to the drug of abuse, a learning known as conditioned place preference. In this paradigm, animals learned to associate the effects of the drug with a specific location and chose to spend time there. The administration of several of these inhibitors after reactivation of the drug-related memory interfered with its reconsolidation and abolished the place preference on later tests. Recently, we also found that disrupting the reconsolidation of a conditioned place preference induced in rats by morphine also leads to a loss of motivational withdrawal evoked in the same place. Interestingly, the hippocampus has a critical role in linking the place preference memory to the context-conditioned withdrawal, as interfering with hippocampal molecular mechanisms after the reactivation of morphine conditioned place preference significantly weakens the motivational withdrawal. Thus, targeting the reconsolidation of memories induced by drugs of abuse may prove to be an important strategy for attenuating context-conditioned withdrawal and, therefore, relapse in opiate addicts (Taubenfeld et al., 2010).

Other studies have investigated a different type of task in which animals form a CS-drug association during drug selfadministration training, a model of drug seeking (Lee et al., 2005, 2006). These experiments showed that infusion of zif268 antisense ODN into the basolateral amygdala before the reactivation of a CS-cocaine association abolishes its impact on the learning of a new cocaine-seeking response or maintenance of cocaine-seeking, as well as relapse to a previously established drug-seeking behavior.
The same group later demonstrated that conditioned withdrawal can be disrupted following the reactivation of a CS-withdrawal association (Hellemans et al., 2006).

From all these investigations, it emerges that finding agents or strategies that disrupt memory reconsolidation is a potentially important approach for developing novel treatments that aim at weakening pathogenic memories. It is important that future studies determine precisely what response is affected by the postreactivation treatments and how the age of the memories changes their sensitivity to treatments.

Finally, one other potentially important clinical application of targeting memory reconsolidation, which has not yet been much explored, is memory enhancement. Can we enhance memory reconsolidation and boost memory retention? Initial findings indicate that memory can be significantly enhanced by targeting mechanisms activated in the post-retrieval phase of memory (Frenkel et al., 2005; Tronson et al., 2006; Chen et al., 2011). This intriguing question requires future exploration and the results thus far obtained imply that, in order to identify memory enhancers, we may need to explore the temporally limited post-training or post-retrieval active phases.

\section{A MODEL TO EXPLAIN MEMORY RECONSOLIDATION}

Memories of a single event that become very long-lasting are evoked by the experience of a very salient or emotional event. Weaker memories require multiple training trials to become very longlasting. Studies of consolidation and reconsolidation, particularly in animal models, have often used models of salient single or few learning trials because they easily allow temporal analysis of the changes that occur after a learning trace is evoked. These studies show that the temporal evolution of the labile consolidation and reconsolidation phases of memory is a function of the intensity of training, reactivations, and the passage of time.

What is memory reconsolidation? What is its function?

Learning of a salient event leads to a long-lasting memory through molecular cellular and circuitry changes that evolve over an extended time (weeks in rats). During the first 1-2 days, memory is disrupted by treatments that interfere with the synthesis of a number of proteins; however, following this time, memory becomes resistant to the same amnestic treatments. This may be interpreted as indicating that consolidation is completed. However, the memory still lies for some time in a sensitive, critical period, during which the memory can again return to a labile state if reactivated for example by retrievals or retraining, and, while in this fragile state, its retention can be bidirectionally modulated. With the passage of time, a gradient of memory stabilization sets in, along with increased resistance to post-retrieval interference.

What is the contribution of time to memory strengthening and consolidation? Various hypotheses are possible. First, the memory trace strengthens because it undergoes implicit reactivations, perhaps as a consequence of rythmicities like circadian rhythm or sleep (Stickgold and Walker, 2007). A salient, aversive or traumatic event is also frequently recalled over and over, especially during the first days or weeks (Rubin et al., 2008). Cues often trigger the retrieval of aversive or traumatic experiences. Perhaps these reactivations, implicit, and explicit, serve the biologically important function of consolidating an aversive memory without repeating the aversive 
experience. Thus, through retrieval or reactivation, reconsolidation strengthens or consolidates important memories. Similarly, multiple trials of weaker learning events would produce the same outcome through rounds of consolidation and reconsolidation. Hence, reconsolidation strengthens and contributes to consolidating the memory.

Reactivation of the memory also has another important function, which is to update our memories and add new information to the past experience. If the information is a repetition of a previous experience, its reactivations may overlap and perhaps even override the network of the original trace and finally lead to strengthening of the same behavior. If the new information encountered is novel and distinct from the recalled experience, the new trace, may become linked to the reactivated trace and create a new, parallel memory that coexists with the original one. Importantly, as this new memory is established via a new consolidation process, new associations can be made even when the old reactivated memory is insensitive to post-reactivation interference (Morris et al., 2006, Winters et al.,

\section{REFERENCES}

Alberini, C. M. (2005). Mechanisms of memory stabilization: are consolidation and reconsolidation similar or distinct processes? Trends Neurosci. 28, 51-56.

Alberini, C. M. (2009). Transcription factors in long-term memory and synaptic plasticity. Physiol. Rev. 89, 121-145.

Alberini, C.M., Milekic, M.H., and Tronel, S. (2006). Mechanisms of memory stabilization and de-stabilization. Cell. Mol. Life Sci. 63, 999-1008.

Bailey, C. H., Kandel, E. R., and Si, K. (2004). The persistence of long-term memory: a molecular approach to self-sustaining changes in learninginduced synaptic growth. Neuron 44 , 49-57.

Bekinschtein, P., Cammarota, M., Igaz, L. M., Bevilaqua, L. R., Izquierdo, I., and Medina, J. H. (2007). Persistence of long-term memory storage requires a late protein synthesis- and BDNFdependent phase in the hippocampus. Neuron 53, 261-277.

Boccia, M. M., Blake, M. G., Acosta, G. B., and Baratti, C. M. (2006). Postretrieval effects of icv infusions of hemicholinium in mice are dependent on the age of the original memory. Learn. Mem. 13, 376-381.

Bramham, C. R., Alme, M. N., Bittins, M., Kuipers, S. D., Nair, R. R., Pai, B., Panja, D., Schubert, M., Soule, J., Tiron, A., and Wibrand, K. (2010). The arc of synaptic memory. Exp. Brain Res. 200, 125-140.

Bustos, S. G., Maldonado, H., and Molina, V. A. (2009). Disruptive effect of midazolam on fear memory reconsolidation: decisive influence of reactivation time span and memory age. Neuropsychopharmacology 34, 446-457.

Cai, W. H., Blundell, J., Han, J., Greene, R. W., and Powell, C. M. (2006).
Postreactivation glucocorticoids impair recall of established fear memory. J. Neurosci. 26, 9560-9566.

Chen, D. Y., Stern, S. A., Garcia-Osta, A., Saunier-Rebori, B., Pollonini, G., Bambah-Mukku, D., Blitzer, R. D., and Alberini, C. M. (2011). A critical role for IGF-II in memory consolidation and enhancement. Nature. 469, 491-497.

Davis, H. P., and Squire, L. R. (1984). Protein synthesis and memory: a review. Psychol. Bull. 96, 518-559.

Davis, S., and Laroche, S. (2006). Mitogenactivated protein kinase/extracellular regulated kinase signalling and memory stabilization: a review. Genes Brain Behav. 5(Suppl. 2), 61-72.

Debiec, J., Doyère, V., Nader, K., and Ledoux, J. E. (2006). Directly reactivated, but not indirectly reactivated, memories undergo reconsolidation in the amygdala. Proc. Natl. Acad. Sci. U.S.A. 103, 3428-3433.

Debiec, J., and LeDoux, J. E. (2004). Disruption of reconsolidation but not consolidation of auditory fear conditioning by noradrenergic blockade in the amygdala. Neuroscience 129, 267-272. (2002). Cellular and systems reconsolidation in the hippocampus. Neuron 36, 527-538.

DeVietti, T. L., Holliday, J.H., and Larson, R. C. (1973). Comparison of amnesias induced by electroconvulsive shock administered after training-trial footshock or noncontingent footshock in rats. J. Comp. Physiol. Psychol. 84, 579-585.

Diergaarde, L., Schoffelmeer, A. N., and De Vries, T. J. (2006). Betaadrenoceptor mediated inhibition of long-term reward-related memory reconsolidation. Behav. Brain Res. 170, 333-336.
Debiec, J., LeDoux, J. E., and Nader, K.

2009). Trace activation and reactivation creates a complex network of stored experiences that is highly dynamic, thus allowing us to adapt to the changing environment. A better understanding of the dynamics of memory consolidation, reconsolidation, and forgetting should help in the design of novel strategies that can either weaken pathogenic memories of memory components or, when advantageous, enhance memory strength.

\section{ACKNOWLEDGMENTS}

Many thanks to all the members of my lab for their invaluable contributions to the work discussed and for their helpful feedback on the manuscript. I also thank Dr. Reginald Miller and the CCMS facility of Mount Sinai for technical support. The work included in this review was supported by the National Institute of Mental Health (R01 MH074736, R01 MH065635), National Institute of Drugs of Abuse (R21 CEBRA DA017672), and the Hirschl and Philoctetes Foundations to Cristina M. Alberini.

Dudai, Y. (2004). The neurobiology of consolidations, or, how stable is the engram? Annu. Rev. Psychol. 55, 51-86.

Dudai, Y., and Eisenberg, M. (2004). Rites of passage of the engram: reconsolidation and the lingering consolidation hypothesis. Neuron 44, 93-100.

Eisenberg, M., and Dudai, Y. (2004). Reconsolidation of fresh, remote, and extinguished fear memory in Medaka: old fears don't die. Eur. J. Neurosci. 20, 3397-3403.

Eisenberg, M., Kobilo, T., Berman, D. E., and Dudai, Y. (2003). Stability of retrieved memory: inverse correlation with trace dominance. Science 301, 1102-1104.

Forcato, C., Rodríguez, M. L., Pedreira, M. E., and Maldonado, H. (2010). Reconsolidation in humans opens up declarative memory to the entrance of new information. Neurobiol. Learn. Mem. 93, 77-84.

Frankland, P. W., and Bontempi, B. (2005). The organization of recent and remote memories. Nat. Rev. Neurosci. 6, 119-130.

Frankland, P. W., Ding, H. K., Takahashi, E., Suzuki, A., Kida, S., and Silva, A. J. (2006). Stability of recent and remote contextual fear memory. Learn. Mem. 13, 451-4577.

Frenkel, L., Maldonado, H., and Delorenzi, A. (2005). Memory strengthening by a real-life episode during reconsolidation: an outcome of water deprivation via brain angiotensin II. Eur. J. Neurosci. 22, 1757-1766.

Fricks-Gleason, A. N., and Marshall, J. F. (2008). Post-retrieval beta-adrenergic receptor blockade: effects on extinction and reconsolidation of cocaine-cue memories. Learn. Mem. 15, 643-648.

García-DeLaTorre, P., Rodriguez-Ortiz, C. J., Arreguin-Martinez, J. L., CruzCastañeda, P., and Bermúdez-Rattoni,
F. (2009). Simultaneous but not independent anisomycin infusions in insular cortex and amygdala hinder stabilization of taste memory when updated. Learn. Mem. 16, 514-519.

Gewirtz, J.C., and Davis, M. (2000). Using pavlovian higher-order conditioning paradigms to investigate the neural substrates of emotional learning and memory. Learn. Mem. 7, 257-266. [Review].

Hellemans, K. G., Everitt, B. J., and Lee, J. L. (2006). Disrupting reconsolidation of conditioned withdrawal memories in the basolateral amygdala reduces suppression of heroin seeking in rats. $J$. Neurosci. 26, 12694-12699.

Hernandez, P. J., Sadeghian, K., and Kelley, A. E. (2002). Early consolidation of instrumental learning requires protein synthesis in the nucleus accumbens. Nat. Neurosci. 5, 1327-1331.

Inda, M.C., Muravieva, E.V., and Alberini, C. M. (2011). Retrieval and the passage of time: from reconsolidation and strengthening to extinction. $J$. Neurosci. 31, 1635-1643.

Kandel, E. R. (2001). The molecular biology of memory storage: a dialog between genes and synapses. Biosci. Rep. 21, 565-611.

Kemenes, G., Kemenes, I., Michel, M., Papp, A., and Muller, U. (2006). Phasedependent molecular requirements for memory reconsolidation: differential roles for protein synthesis and protein kinase A activity. J. Neurosci. 26, 6298-6302.

Kindt, M., Soeter, M., and Vervliet, B. (2009). Beyond extinction: erasing human fear responses and preventing the return of fear. Nat. Neurosci. 12, 256-258.

Lee, J. L. (2008). Memory reconsolidation mediates the strengthening of memories by additional learning. Nat. Neurosci. 11, 1264-1266. 
Lee, J. L., Di Ciano, P., Thomas, K. L., and Everitt, B. L. (2005). Disrupting reconsolidation of drug memories reduces cocaine-seeking behavior. Neuron 47 , 795-801.

Lee, J. L., and Everitt, B. J. (2008). Reactivation-dependent amnesia in Pavlovian approach and instrumental transfer. Learn. Mem. 15, 597-602.

Lee, J. L., Everitt, B. J., and Thomas, K. L. (2004). Independent cellular processes for hippocampal memory consolidation and reconsolidation. Science 304, 839-843.

Lee, J. L., Milton, A. L., and Everitt, B. J. (2006). Cue-induced cocaine seeking and relapse are reduced by disruption of drug memory reconsolidation. $J$. Neurosci. 26, 5881-5587.

Lewis, D. J. (1979). Psychobiology of active and inactive memory. Psychol. Bull. 86, 1054-1083.

Litvin, O. O., and Anokhin, K. V. (2000). Mechanisms of memory reorganization during retrieval of acquired behavioral experience in chicks: the effects of protein synthesis inhibition in the brain. Neurosci. Behav. Physiol. 30, 671-678.

Luft, A. R., Buitrago, M. M., Ringer, T., Dichgans, J., and Schulz, J. B. (2004). Motor skill learning depends on protein synthesis in motor cortex after training. J. Neurosci. 24, 6515-6520.

McGaugh, J. L. (2000). Memory - a century of consolidation. Science 287, 248-251

McGaugh, J. L., and Roozendaal, B. (2002). Role of adrenal stress hormones in forming lasting memories in the brain. Curr. Opin. Neurobiol. 2, 205-210.

Meiri, N., and Rosenblum, K. (1998). Lateral ventricle injection of the protein synthesis inhibitor anisomycin impairs long-term memory in a spatial memory task. Brain Res. 789, 48-55.

Melchor, J. P., and Strickland, S. (2005). Tissue plasminogen activator in central nervous system physiology and pathology. Thromb. Haemost. 93, 655-660.

Milekic, M.H., and Alberini, C. M. (2002). Temporally graded requirement for protein synthesis following memory reactivation. Neuron 36, 521-525.

Milekic, M. H., Brown, S. D., Castellini, C., and Alberini, C. M. (2006). Persistent disruption of an established morphine conditioned place preference. J. Neurosci. 26, 3010-3020.

Miller, C. A., and Marshall, J. F. (2005). Molecular substrates for retrieval and reconsolidation of cocaine-associated contextual memory. Neuron 47 , 873-884.

Milton, A. L., Lee, J. L., and Everitt, B. J. (2008). Reconsolidation of appetitive memories for both natural and drug reinforcement is dependent on $\{$ beta $\}$ adrenergic receptors. Learn. Mem. 15, 88-92.

Monfils, M. H., Cowansage, K. K., Klann, E., and LeDoux, J. E. (2009). Extinction-reconsolidation boundaries: key to persistent attenuation of fear memories. Science 324, 951-955.

Morris, R. G., Inglis, J., Ainge, J. A., Olverman, H. J., Tulloch, J., Dudai, Y., and Kelly, P. A. (2006). Memory reconsolidation: sensitivity of spatial memory to inhibition of protein synthesis in dorsal hippocampus during encoding and retrieval. Neuron 50, 479-489.

Moscovitch,M., Rosenbaum, R. S., Gilboa, A., Addis, D. R., Westmacott, R., Grady, C., McAndrews, M. P., Levine, B., Black, S., Winocur, G., and Nadel, L. (2005). Functional neuroanatomy of remote episodic, semantic and spatial memory: a unified account based on multiple trace theory. J. Anat. 207, 35-66. [Review].

Muller, G. E., and Pilzecker, A. (1900). Experimentelle Beitrage zur Lehre von Gedächtnis. Z. Psychol. 1, 1-300.

Muravieva, E. V., and Alberini, C. M. (2010). Limited efficacy of propranolol on the reconsolidation of fear memories. Learn. Mem. 17, 306-313.

Nadel, L., and Moscovitch, M. (1997). Memory consolidation, retrograde amnesia and the hippocampal complex. Curr. Opin. Neurobiol. 7, 217-227.

Nader, K., and Einarsson, E. O. (2010). Memory reconsolidation: an update. Ann. N. Y. Acad. Sci. 1191, 27-41. [Review].

Nader, K., Schafe, G. E., and LeDoux, J. E. (2000a). The labile nature of consolidation theory. Nat. Rev. Neurosci. 1,216-219. [Review].

Nader, K., Schafe, G. E., and Le Doux, J. E. (2000b). Fear memories require protein synthesis in the amygdala for reconsolidation after retrieval. Nature 406, 722-726.

O'Brien, C. P., Childress, A. R., McLellan, A. T., and Ehrman, R. (1992). Classical conditioning in drug-dependent humans. Ann. N. Y. Acad. Sci. 654, 400-415.

O'Donnell, T., Hegadoren, K. M., and Coupland,N.C. (2004). Noradrenergic mechanisms in the pathophysiology of post-traumatic stress disorder. Neuropsychobiology 50, 273-283.

Ou, L. C., Yeh, S. H., and Gean, P. W. (2010). Late expression of brainderived neurotrophic factor in the amygdala is required for persistence of fear memory. Neurobiol. Learn. Mem. $93,372-382$.

Parvez, K., Moisseev, V., and Lukowiak, K. (2006). A context-specific single contingent-reinforcing stimulus boosts intermediate-term memory into long-term memory. Eur. J. Neurosci. 24, 606-616.

Pitman, R. K. (1989). Post-traumatic stress disorder, hormones, and memory. Biol. Psychiatry 26, 221-223.

Pitman, R. K., and Delahanty, D. L. (2005). Conceptually driven pharmacologic approaches to acute trauma. CNS Spectr. 2, 99-106.

Przybyslawski, J., Roullet, P., and Sara, S. J. (1999). Attenuation of emotional and nonemotional memories after their reactivation: role of beta adrenergic receptors. J. Neurosci. 19, 6623-6628.

Reissner, K. J., Shobe, J. L., and Carew, T. J. (2006). Molecular nodes in memory processing: insights from Aplysia. Cell. Mol. Life Sci. 63, 963-974.

Rescorla, R. A. (1980). Pavlovian SecondOrder Conditioning. Studies in Associative Learning. Hillsdale, NJ: Lawrence Erlbaum Associates), 120.

Robinson, M. J., and Franklin, K. B. (2010). Reconsolidation of a morphine place preference: impact of the strength and age of memory on disruption by propranolol and midazolam. Behav. Brain. Res. 213, 201-207.

Rodriguez-Ortiz, C. J., De la Cruz, V., Gutierrez, R., and Bermudez-Rattoni, F. (2005). Protein synthesis underlies post-retrieval memory consolidation to a restricted degree only when updated information is obtained. Learn. Mem. 12, 533-537.

Rodriguez-Ortiz, C. J., Garcia-DeLaTorre, P., Benavidez, E., Ballesteros, M. A. and Bermudez-Rattoni, F. (2008). Intrahippocampal anisomycin infusions disrupt previously consolidated spatial memory only when memory is updated. Neurobiol. Learn. Mem. 89, 352-359.

Roozendaal, B. (2002). Stress and memory: opposing effects of glucocorticoids on memory consolidation and memory retrieval. Neurobiol. Learn. Mem. 3, 578-595.

Rossato, J. I., Bevilaqua, L. R., Medina, J. H., Izquierdo, I., and Cammarota M. (2006). Retrieval induces hippocampal-dependent reconsolidation of spatial memory. Learn. Mem. 13 , 431-440.

Rubin, D. C., Boals, A., and Berntsen, D. (2008). Memory in posttraumatic stress disorder: properties of voluntary and involuntary, traumatic and nontraumatic autobiographical memories in people with and without posttraumatic stress disorder symptoms. J. Exp. Psychol. Gen. 137, 591-614.

Sara, S. J. (2000a). Retrieval and reconsolidation: toward a neurobiology of remembering. Learn. Mem. 7, 73-84. [Review].

Sara, S. J. (2000b). Strengthening the shaky trace through retrieval. Nat. Rev. Neurosci. 1, 212-213.
Schiller, D., Monfils, M. H., Raio, C. M. Johnson, D. C., Ledoux, J. E., and Phelps, E. A. (2010). Preventing the return of fear in humans using reconsolidation update mechanisms. Nature 463, 49-53.

Smith, C. N., and Squire, L. R. (2009). Medial temporal lobe activity during retrieval of semantic memory is related to the age of the memory. $J$. Neurosci. 29, 930-938.

Squire, L. R. (2009). Memory and brain systems: 1969-2009. J. Neurosci. 29, 12711-12716.

Squire, L. R., and Alvarez, P. (1995) Retrograde amnesia and memory consolidation: a neurobiological perspective. Curr. Opin. Neurobiol. 5, 169-177.

Stickgold, R., and Walker, M. P. (2007). Sleep-dependent memory consolidation and reconsolidation. Sleep Med. 8, 331-343.

Suárez, L. D., Smal, L., and Delorenzi A. (2010). Updating contextual information during consolidation as result of a new memory trace. Neurobiol. Learn. Mem. 93, 561-571.

Suzuki, A., Josselyn, S. A., Frankland, P.W., Masushige, S., Silva, A. J., and Kida, S. (2004). Memory reconsolidation and extinction have distinct temporal and biochemical signatures. J. Neurosci. 24 , 4787-4795.

Takehara-Nishiuchi,K., and McNaughton, B. L. (2008). Spontaneous changes of neocortical code for associative memory during consolidation. Science 322 , 960-963.

Taubenfeld, S. M., Milekic, M. H., Monti, B., and Alberini, C. M. (2001a). The consolidation of new but not reactivated memory requires hippocampal C/EBPß. Nat. Neurosci. 4, 813-818.

Taubenfeld, S. M., Wiig, K. A., Monti, B., Dolan, B., Pollonini, G., and Alberini, C. M. (2001b). Fornix-dependent induction of hippocampal CCAAT enhancer-binding protein [beta] and [delta] Co-localizes with phosphorylated cAMP response elementbinding protein and accompanies long-term memory consolidation. $J$. Neurosci. 21, 84-91.

Taubenfeld, S. M., Muravieva, E., GarciaOsta, A., and Alberini, C. M. (2010). Disrupting the memory of places induced by drugs of abuse weakens withdrawal in a context-dependent manner: critical role of the hippocampus. Proc. Natl. Acad. Sci. U.S.A. 107, 12345-12350.

Taubenfeld, S. M., Riceberg, J. S., New, A. S., and Alberini, C. M. (2009). Preclinical assessment for selectively disrupting a traumatic memory via postretrieval inhibition of glucocorticoid receptors. Biol. Psychiatry 65, 249-257. 
Tollenaar, M. S., Elzinga, B. M., Spinhoven, P., and Everaerd, W. (2009). Immediate and prolonged effects of cortisol, but not propranolol, on memory retrieval in healthy young men. Neurobiol. Learn. Mem. 91, 23-31.

Touzani, K., Puthanveettil, S. V., and Kandel, E. R. (2007). Consolidation of learning strategies during spatial working memory task requires protein synthesis in the prefrontal cortex. Proc. Natl. Acad. Sci. U.S.A. 104, 5632-5637.

Tronel, S., and Alberini, C. M. (2007). Persistent disruption of a traumatic memory by post-retrieval inactivation of glucocorticoid receptors in the amygdala. Biol. Psychiatry 62, 33-39.

Tronel, S., Milekic, M. H., and Alberini, C. M. (2005). Linking new information to a reactivated memory requires consolidation and not reconsolidation mechanisms. PLoS Biol. 3, 1630-1638. doi: 10.1371/journal.pbio.0030293
Tronson, N. C., and Taylor, J. R. (2007) Molecular mechanisms of memory reconsolidation. Nat. Rev. Neurosci. 8, 262-275.

Tronson, N. C, Wiseman, S. L. Olausson, P., and Taylor, J. R. (2006). Bidirectional behavioral plasticity of memory reconsolidation depends on amygdalar protein kinase A. Nat. Neurosci. 9, 167-169.

Valjent, E., Corbille, A. G., BertranGonzalez, J., Herve, D., and Girault, J. A. (2006). Inhibition of ERK pathway or protein synthesis during reexposure to drugs of abuse erases previously learned place preference. Proc. Natl. Acad. Sci. U.S.A. 103, 2932-2937.

von Hertzen, L. S., and Giese, K. P. (2005). Memory reconsolidation engages only a subset of immediate-early genes induced during consolidation. J. Neurosci. 25, 1935-1942.

Wang, H., Hu, Y., and Tsien, J. Z. (2006). Molecular and systems mechanisms of memory consolidation and storage. Prog. Neurobiol. 79, 123-135.

Winocur, G., Moscovitch, M., and Bontempi, B. (2010). Memory formation and long-term retention in humans and animals: convergence towards a transformation accoun of hippocampal-neocortical interactions. Neuropsychologia. 48 , 2339-2356.

Winocur, G., Moscovitch, M., and Sekeres, M. (2007). Memory consolidation or transformation: context manipulation and hippocampal representations of memory. Nat. Neurosci. 10, 555-557.

Winters, B. D., Tucci, M.C., and DaCostaFurtado, M. (2009). Older and stronger object memories are selectively destabilized by reactivation in the presence of new information. Learn. Mem. 16, 545-553.

Yehuda, R. (2006). Advances in understanding neuroendocrine alterations in PTSD and their therapeutic impli- cations. Ann. N. Y. Acad. Sci. 1071, 137-166.

Conflict of Interest Statement: The author declares that the research was conducted in the absence of any commercial or financial relationships that could be construed as a potential conflict of interest.

Received: 29 July 2010; accepted: 23 February 2011; published online: 07 March 2011.

Citation: Alberini CM (2011) The role of reconsolidation and the dynamic process of long-term memory formation and storage. Front. Behav. Neurosci. 5:12. 10.3389/ fnbeh.2011.00012

Copyright (c) 2011 Alberini. This is an open-access article subject to an exclusive license agreement between the authors and Frontiers Media SA, which permits unrestricted use, distribution, and reproduction in any medium, provided the original authors and source are credited. 